\title{
Treatment of warts with topical curettage and PDL
}

\begin{abstract}
Cutaneous warts are benign epidermal proliferations caused by human Papilloma virus infection. The treatment is challenging despite the fact that there are several treatments because any therapy was not established as gold standard. Although therapeutic abstinence is sometimes advisable, more patients request active treatment because of social stigma or discomfort. More active treatments have specific disadvantages and side effects. Some studies have used pulsed dye lasers to treat cutaneous warts and a recent review noted a very large ranging of rate response. In this report we presented a case of successfully removed resistant plantar warts using 3 sessions of pulsed dye laser associated with a topical curettage.
\end{abstract}

Keywords: epithelial neoplasms, skin, photodermal, cryotherapy sessions, PDL
Volume 2 Issue I - 2018

\section{Salama Hegazy, Maria Polina konstantinou, Carle Paul, Cristina Bulai Livideanu}

Department of Dermatology, Paul Sabatier University, France

\section{Correspondence: Salama Hegazy, Department of} Dermatology, Paul Sabatier University and Toulouse University Hospital,Toulouse, France, Email dr_salama_86@hotmail.com Received: December 08, 2017 | Published: January 24,
2018

\section{Introduction}

Warts are benign epithelial neoplasms of the skin and mucosa resulting from human Papilloma virus (HPV) infection. They are a common dermatologic complaint, with an estimated incidence of $10 \%$ in children and young adults. ${ }^{1}$ The treatment is challenging and there is no single therapy established as completely curative. ${ }^{2}$ In this report we presented a case of successfully removed resistant plantar warts using pulsed dye laser associated with chemical and mechanic curettage.

\section{Case presentation}

A 70 years man, without immunosuppression history, has consulted to our department with two large mosaic warts on the feet (Figure 1A). This lesion persisted for several years in despite of a performed treatment with many cryotherapy sessions (Figure 1B). We proposed a treatment combining 3 sessions of pulsed dye laser (PDL) with chemical and mechanic curettage. Prior to every laser sessions, warts were treated with topical keratolytic (a combination of $16 \%$ lactic and 16\% salicylic acids in collodion base) and were peeled with a surgical blade (Figure 1C). The following parameters were used: fluency of $10 \mathrm{~J} / \mathrm{cm}^{2}$, pulse duration of $0.5 \mathrm{~ms}$, and a spot size of $7 \mathrm{~mm}$. Two slightly overlapping passes were applied per each wart and 1-mm margin on the surrounding skin. Standardized air-cooling (Zimmer air cooler) was administered during treatment for thermal protection of the epidermis. An immediate purpuric endpoint is achieved (Figure 1D).
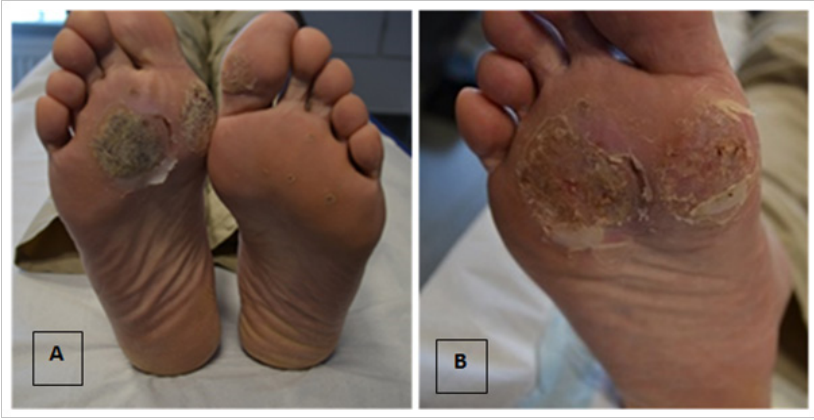
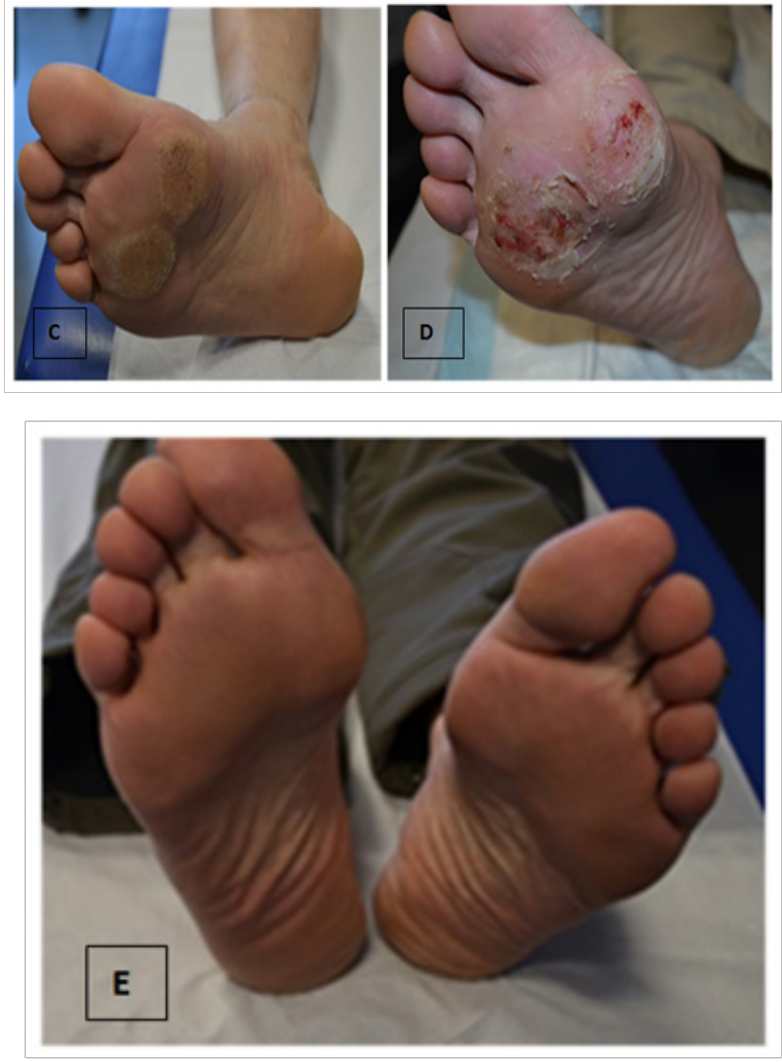

Figure I

(A) LARGE resistant mosaic wart on the feet before treatment;

(B) Clinical aspect after curettage with surgical blade and topical keratolytic (right foot);

(C) Endpoint achieved after the first session with PDL (right foot);

(D) Partial remission after the second session of PDL (right foot);

(E) Patient's feet are clear of the wart at one month follow-up after the third session. 
After 3 sessions at 1-month intervals the feet were totally cleared of the wart. The patient tolerated the treatment well and there were no scars or other adverse effects. The patient's status was checked one month after the third session, the feet were still clear and there was no sign of recurrence of the wart (Figure 1E).

\section{Discussion}

Here we reported a case of resistant large mosaic warts on the feet treated efficacy and safe with associated topical curettage and PDL treatment. No recurrence was noted to one months of follow-up. Several treatments are available for treatment of warts without gold standard established therapy neither for disappearance or to prevent recurrence. ${ }^{3}$ Most therapeutic methods have specific disadvantages and side effects as pain and long recovery periods. Topical management requires the application of topical drugs for long durations and treatment success is highly dependent on patient compliance. Ideally, treatment should be simple with a low risk of adverse effects. ${ }^{4,5}$ Many studies have used 585-nm PDL with the wart blood vessels as the target tissue. ${ }^{6}$ As the hemoglobin heats up, thermal energy is dissipated to surrounding tissues, leading to cauterization of blood vessels (photo dermal or photomechanical destruction). ${ }^{7}$ This is because hemoglobin in blood has strong absorption peaks at wavelengths ranging from 585 to $595 \mathrm{~nm}){ }^{8}$ In literature data, the response rates seen in peripheral warts (involving the hands and feet) were very variable, ranging between $48 \%$ and $95 \% .^{9}$ This large variation could be linked by the ability of the laser waves to penetration in papillary dermis-to destroy blood vessels-fact probably conditioned by thickness of the epidermis. Chemical and mechanical curettages that we used in our patient play an important role in the penetration of laser waves.

\section{Conclusion}

PDL associated with chemical and mechanical curettage is an effective, safe and well tolerated treatment for resistant warts.

\section{Acknowledgements}

None.

\section{Conflicts of interest}

The author declares no conflicts of interest.

\section{References}

1. Robson KJ, Cunningham NM, Kruzan KL, et al. Pulsed-dye laser versus conventional therapy in the treatment of warts: a prospective randomized trial. J Am Acad Dermatol. 2000;43(2 Pt 1):275-280.

2. Micali G, Dall'Oglio F, Nasca MR, et al. Management of cutaneous warts: an evidence-based approach. Am J Clin Dermatol. 2004;5(5):311-317.

3. Loo SK, Tang WY. Warts (non-genital). BMJ Clin Evid. 2014.

4. Mulhem E, Pinelis S. Treatment of nongenital cutaneous warts. Am Fam Physician. 2011;84(3):288-293.

5. Schellhaas U, Gerber W, Hammes S, et al. Pulsed dye laser treatment is effective in the treatment of recalcitrant viral warts. Dermatol Surg. 2008;34(1):67-72.

6. Veitch D, Kravvas G, Al-Niaimi F. Pulsed dye laser therapy in the treatment of warts: a review of the literature. Dermatol Surg. 2017;43(4):485-493.

7. Wu C, Langan S, Kilmurray M, et al. Efficacy of pulsed-dye laser for viral warts--an internal audit. Ir Med J. 2003;96(3):82-83.

8. Kopera D. Verrucae vulgares: flashlamp-pumped pulsed dye laser treatment in 134 patients. Int J Dermatol. 2003;42(11):905-908.

9. Nguyen J, Korta DZ, Chapman LW, et al. Laser treatment of nongenital verrucae: a systematic review. JAMA Dermatol. 2016;152(9):1025-1034. 